\title{
Exact ground states of quantum many-body systems under confinement
}

\author{
Adolfo del Campo (1) \\ Donostia International Physics Center, E-20018 San Sebastián, Spain; \\ IKERBASQUE, Basque Foundation for Science, E-48013 Bilbao, Spain; \\ Department of Physics, University of Massachusetts, Boston, Massachusetts 02125, USA; \\ and Theory Division, Los Alamos National Laboratory, MS-B213, Los Alamos, New Mexico 87545, USA
}

(Received 12 May 2020; accepted 2 October 2020; published 21 October 2020)

\begin{abstract}
Knowledge of the ground state of a homogeneous quantum many-body system can be used to find the exact ground state of a dual inhomogeneous system with a confining potential. We first identify the complete family of one-dimensional parent Hamiltonians with a ground state of Bijl-Jastrow form in free space. For each instance in this family, the dual trapped system is shown to include a one-body harmonic potential and two-body long-range interactions. The extension to anharmonic potentials and quantum solids with Nosanov-Jastrow ground-state wave functions is also presented. We apply this exact mapping to construct eigenstates of trapped systems from free-space solutions for different choices of the pair correlation functions entering the Bijl-Jastrow form. This construction is user friendly and allows one to readily rederive known instances of quasisolvable and integrable models, as well as to introduce unexplored examples. Among the latter, we describe a Lieb-Liniger-Coulomb model whose exact ground state is a McGuire soliton solution embedded in a harmonic trap.
\end{abstract}

DOI: 10.1103/PhysRevResearch.2.043114

\section{INTRODUCTION}

Exact solutions play an important role in physics. Solvable models often bring novel insights, they can serve as a test bed for physical theories and a starting point for new approximations, and help benchmarking numerical methods. Certain solvable models are known to be integrable. In the classical domain, this requires the existence of a number of conserved quantities equal to (or even greater than) that of degrees of freedom. In the quantum domain, integrability is often associated with scattering without diffraction, encoded in the Yang-Baxter equations [1]. Quantum integrable systems are generally homogeneous. For quantum many-body systems of continuous variables (e.g., those describing quantum gases and liquids), known exact solutions are typically associated with the absence of an external potential. In the one-dimensional case, an exact treatment is possible via the Bethe ansatz approach, which expresses the wave function as a superposition of plane waves [2-4]. This reduces the possible settings to free space (no external one-body potential), translationally invariant ring geometries with periodic boundary conditions, or settings with hard walls such as mirrors and boxlike traps $[5,6]$.

Known solutions for systems in the presence of an external confining potential that varies gradually in space are rare. Among them, the most prominent case is perhaps that of the

Published by the American Physical Society under the terms of the Creative Commons Attribution 4.0 International license. Further distribution of this work must maintain attribution to the author(s) and the published article's title, journal citation, and DOI. rational Calogero-Sutherland gas, describing one-dimensional bosons with inverse-square interactions in a harmonic trap $[1,7,8]$. The latter includes as well hard-core bosons, the socalled Tonks-Girardeau gas, which was first studied in the continuum [9] and then in a harmonic trap [10] (in addition to ring and boxlike traps). While some tools, such as the Bose-Fermi duality and anyon-fermion mapping [11,12] or the generalizations to mixtures [13,14], increase the variety of models, these can be considered all part of the same family.

In the study of nonlinear physics, variants of integrable solutions in free space have been found in inhomogeneous systems. However, as pointed out by Kundu, these are related to homogeneous systems via gauge, scaling, and coordinate transformations [15]. An analog approach has proved successful in the study of ultracold fermions in the unitary limit [16]. In this context, it has been possible to relate universal zero-energy states in free space, exhibiting scale invariance, with eigenstates of the same system embedded in a harmonic trap [17-19].

Knowledge of the ground state of a many-body quantum system is of great importance, as its structure determines the nature of low-lying excitations built upon it. In addition, as the ground state includes important correlations among particles, it may be the case that excitations are free (see, e.g., Refs. [1,20-22]). In some cases, the ground state determines the structure of the complete spectrum, as in the Calogero-Sutherland gas [22-26]. In quasisolvable models, knowledge of the ground state allows one to construct towers of excited states that span part of the spectrum. An example of this type is the Jain-Khare model involving bosons with two-body and three-body inverse-square interactions among nearest and next-nearest neighbors, respectively [27-31]. The same holds true for the truncated Calogero-Sutherland 
gas with interactions restricted to a number of neighbors $[32,33]$.

In this paper, we introduce a general construction to find quasisolvable models describing particles confined in a harmonic trap and subject to long-range pairwise interactions. We find the complete family of models describing indistinguishable bosons in a harmonic trapped with a ground state of Bijl-Jastrow form. This construction is then generalized to anharmonic external potentials and the description of quantum solids with ground-state wave functions of Nosanov-Jastrow form.

This user-friendly approach works as a factory of quasisolvable models. Given a choice of the pair correlation function entering the ground-state wave function, one needs only to compute its first and second derivatives. With this information at hand, one readily finds the following: (i) the parent Hamiltonian in free space, which generally includes two-body and three-body interactions and has a vanishing ground-state energy eigenvalue; (ii) the parent Hamiltonian of the trapped system, (iii) its wave function, and (iv) energy eigenvalue; and (v) the parent Hamiltonian of the corresponding quantum solid, (vi) its ground-state wave function, and (vii) its energy eigenvalue.

The power of our approach to identify unexplored (quasi)solvable and (quasi)integrable models is unveiled by different choices of the pair correlation functions entering the ground-state wave function. Among them, we describe the Lieb-Liniger-Coulomb model with a trapped McGuire soliton as an exact ground state and a trapped system with Toda-Like pairwise interactions.

\section{AN EXACT MAPPING}

Consider a many-body problem in the absence of a trapping potential and satisfying a time-independent Schrödinger equation,

$$
\hat{H}_{0}\left|\Phi_{n}\right\rangle=E_{n}\left|\Phi_{n}\right\rangle,
$$

with a generic many-body Hamiltonian in one spatial dimension,

$\hat{H}_{0}=-\frac{\hbar^{2}}{2 m} \sum_{i=1}^{N} \frac{\partial^{2}}{\partial x_{i}^{2}}+\sum_{i<j} v\left(x_{i}, x_{j}\right)+\sum_{i<j<k} w\left(x_{i}, x_{j}, x_{k}\right)+\cdots$,

in which $v(x, y)$ accounts for the two-body interactions, $w(x, y, z)$ for three-body interactions, and so on. In particular, we denote the wave function of the ground state of $\hat{H}_{0}$ by $\Phi_{0}$ and its eigenenergy by $E_{0}$.

We are interested in the embedding of this system in a harmonic trap so that the total Hamiltonian reads

$$
\hat{H}=\hat{H}_{0}+\sum_{i=1}^{N} \frac{1}{2} m \omega^{2} x_{i}^{2}+V\left(x_{1}, \ldots, x_{N}\right),
$$

where $V\left(x_{1}, \ldots, x_{N}\right)$ is an additional interaction potential that will be required for consistency. As an ansatz for the ground state in the presence of the harmonic trap we try the wave function

$$
\Psi_{0}\left(x_{1}, \ldots, x_{N}\right)=\exp \left(-\frac{m \omega}{2 \hbar} \sum_{i=1}^{N} x_{i}^{2}\right) \Phi_{0}\left(x_{1}, \ldots, x_{N}\right) .
$$

To derive the parent Hamiltonian (2), we compute the action of the kinetic energy operator on $\Psi_{0}\left(x_{1}, \ldots, x_{N}\right)$. It is not difficult to show that

$$
\begin{aligned}
& {\left[\hat{H}_{0}+\sum_{i=1}^{N} \frac{1}{2} m \omega^{2} x_{i}^{2}\right] \Psi_{0}-\hbar \omega e^{-\frac{m \omega}{2 \hbar} \sum_{i=1}^{N} x_{i}^{2}} \sum_{i=1}^{N} x_{i} \partial_{x_{i}} \Phi_{0}} \\
& \quad=\left(E_{0}+\frac{N \hbar \omega}{2}\right) \Psi_{0} .
\end{aligned}
$$

We are interested in rewriting this equation as a standard many-body Schrödinger equation, without velocity-dependent interactions. As we shall see, this is possible in a number of cases. For instance, it is known that universal zero-energy states $\Psi_{0}$ exhibiting scale invariance are eigenstates of the dilatation operator $\hat{D}=\sum_{i=1}^{N} x_{i} \partial_{x_{i}}$, satisfying $\hat{D} \Psi_{0}=\nu \Psi_{0}$ with eigenvalue $v$. In such a case, Eq. (4) does indeed reduce to a many-body Schrödinger equation. This is the strategy used to find eigenstates of a unitary Fermi gas in a three-dimensional harmonic trap [17-19].

In what follows, we shall consider the ground state of $\hat{H}_{0}$ to be of Bijl-Jastrow form,

$$
\Phi_{0}\left(x_{1}, \ldots, x_{N}\right)=\left\langle x_{1}, \ldots, x_{N} \mid \Phi_{0}\right\rangle=\prod_{i<j} f\left(x_{i j}\right),
$$

a product of a single pair correlation function $f\left(x_{i j}\right)$ over each pair of particles [1]. Here, we denote the interparticle distance by $x_{i j}=x_{i}-x_{j}$ and note that $f\left(x_{i j}\right)=\epsilon f\left(-x_{i j}\right)$ with $\epsilon=1$ for bosons and $\epsilon=-1$ for fermions. (It is actually possibly to consider a more general case of one-dimensional anyons $[11,34,35])$.

In this case, the parent Hamiltonian $\hat{H}_{0}$ involves exclusively two-body and three-body interactions

$$
\hat{H}_{0}=-\frac{\hbar^{2}}{2 m} \sum_{i=1}^{N} \frac{\partial^{2}}{\partial x_{i}^{2}}+V_{2}+V_{3},
$$

where the two-body and three-body potentials are given by

$$
\begin{gathered}
V_{2}=\frac{\hbar^{2}}{m} \sum_{i<j} \frac{f^{\prime \prime}\left(x_{i j}\right)}{f\left(x_{i j}\right)}, \\
V_{3}=\frac{\hbar^{2}}{m} \sum_{i<j<k}\left[\frac{f^{\prime}\left(x_{i j}\right) f^{\prime}\left(x_{i k}\right)}{f\left(x_{i j}\right) f\left(x_{i k}\right)}-\frac{f^{\prime}\left(x_{i j}\right) f^{\prime}\left(x_{j k}\right)}{f\left(x_{i j}\right) f\left(x_{j k}\right)}\right. \\
\left.+\frac{f^{\prime}\left(x_{i k}\right) f^{\prime}\left(x_{j k}\right)}{f\left(x_{i k}\right) f\left(x_{j k}\right)}\right] .
\end{gathered}
$$

Here, $f^{\prime}$ and $f^{\prime \prime}$ denote the first and second spatial derivatives of $f$, respectively. This result provides the complete family of one-dimensional parent Hamiltonians with a ground state of Bijl-Jastrow form in free space. It is similar in spirit to that found by Calogero and Marchioro in three-dimensional systems [36]. However, by focusing on one spatial dimension, both $V_{2}$ and $V_{3}$ take a simpler form.

Under periodic boundary conditions, the family of Hamiltonians $\hat{H}_{0}$ that involve exclusively two-body interactions has been studied in detail [1]. We shall focus instead on systems in the real line, of relevance to the description of ultracold gases in tight waveguides. As we shall see, $V_{3}$ effectively vanishes in a number of relevant examples. 
Using (5)-(8) we note that the energy eigenvalue of $\Phi_{0}$ is $E_{0}=0$, e.g.,

$$
\hat{H}_{0} \Phi_{0}=0 .
$$

In what follows, we consider the ground state in the presence of a harmonic trap to be of the form

$$
\Psi_{0}=\exp \left(-\frac{m \omega}{2 \hbar} \sum_{i=1}^{N} x_{i}^{2}\right) \prod_{i<j} f\left(x_{i j}\right) .
$$

By explicit computation, one then finds

$$
e^{-\frac{m \omega}{2 \hbar} \sum_{i=1}^{N} x_{i}^{2}} \sum_{i=1}^{N} x_{i} \partial_{x_{i}} \prod_{i<j} f\left(x_{i j}\right)=\sum_{i<j}^{N} x_{i j} \frac{f^{\prime}\left(x_{i j}\right)}{f\left(x_{i j}\right)} \Psi_{0},
$$

which yields the following many-body Schrödinger equation,

$$
\hat{H} \Psi_{0}=\left[\hat{H}_{0}+\sum_{i=1}^{N} \frac{1}{2} m \omega^{2} x_{i}^{2}+V_{2 \mathrm{~L}}\right] \Psi_{0}=\frac{N \hbar \omega}{2} \Psi_{0},
$$

where we have identified the long-range two-body potential

$$
V_{2 \mathrm{~L}}=-\hbar \omega \sum_{i<j}^{N} x_{i j} \frac{f^{\prime}\left(x_{i j}\right)}{f\left(x_{i j}\right)} .
$$

Equations (12) and (13), with $\hat{H}_{0}$ given by Eqs. (6)-(8), provide the complete family of parent Hamiltonians whose exact ground state is of Bijl-Jastrow form and given by (3), describing harmonically trapped quantum particles subject to two-body and possibly three-body interactions. This is our main result, that we next apply to derive a number of exact many-body solutions, with a focus on cases in which $V_{3}$ is constant, and thus simply contribute to the energy eigenvalue $\mathcal{E}_{0}$ of $\Psi_{0}$.

\section{CALOGERO-SUTHERLAND GAS}

Let us first consider the rational Calogero-Sutherland model $[7,8]$ describing trapped bosonic particles with inversesquare interactions. This model describes free bosons in one dimension for $\lambda=0$ and hard-core bosons in the TonksGirardeau regime for $\lambda=1$ [9], a regime experimentally explored in Refs. [37,38]. For arbitrary $\lambda$, the CalogeroSutherland model can be considered as an ideal gas of particles obeying generalized exclusion statistics [39,40], as shown by Murthy and Shankar [41]. In the absence of the trapping potential, $\hat{H}_{0}$ describes the Calogero-Moser model, with a ground-state wave function $\Phi_{0}=\prod_{i<j}\left|x_{i j}\right|^{\lambda}$ and energy eigenvalue $E_{0}=0$. Indeed, for

$$
f\left(x_{i j}\right)=\left|x_{i j}\right|^{\lambda},
$$

Eq. (7) reduces to

$$
V_{2}=\frac{\hbar^{2}}{m} \lambda(\lambda-1) /\left|x_{i j}\right|^{2},
$$

while the three-body term (8) identically vanishes,

$$
V_{3}=0 .
$$

In the presence of the trap, we consider

$$
\Psi_{0}=\exp \left(-\frac{m \omega}{2 \hbar} \sum_{i=1}^{N} x_{i}^{2}\right) \prod_{i<j}\left|x_{i j}\right|^{\lambda} .
$$

In this case, the long-range two-body potential reads

$$
V_{2 \mathrm{~L}}=-\hbar \omega \lambda \frac{N(N-1)}{2},
$$

and is thus a constant, resembling a mean-field energy contribution to the ground-state energy. As a result, $\Psi_{0}$ has energy $\mathcal{E}_{0}=\frac{\hbar \omega}{2} N[1+\lambda(N-1)]$ which reproduces precisely the known expression for the ground-state energy for the (rational) Calogero-Sutherland gas in a harmonic trap [8]. As a particular case, for $\lambda=1, \Psi_{0}$ and $\mathcal{E}_{0}$ match the corresponding results for the harmonically trapped Tonks-Girardeau gas [10].

\section{LIEB-LINIGER AND LIEB-LINIGER-COULOMB GASES}

The Lieb-Liniger model with contact interactions described by a Dirac delta function $[42,43]$ occupies a unique status among integrable systems, being both Bethe ansatz solvable and directly relevant to the description of ultracold gases in tight waveguides [44]. In homogeneous space, the model supports both bright [45] and dark [46-48] quantum many-body solitons. Let us consider the wave function $\Phi_{0}=\exp \left(g \sum_{i<j}\left|x_{i j}\right|\right)$ where $g$ is a coupling constant. This choice is motivated by the fact that for $g<0, \Phi_{0}$ describes a McGuire bright quantum many-body soliton which is an energy eigenstate [45]. We note that the logarithmic spatial derivative of the pair function

$$
f\left(x_{i j}\right)=\exp \left(g\left|x_{i j}\right|\right)
$$

is given in terms of the sign function $f^{\prime}\left(x_{i j}\right) / f\left(x_{i j}\right)=$ $g \operatorname{sgn}\left(x_{i j}\right)$. Writing the latter in terms of the Heaviside step function $\Theta(x)$ as $\operatorname{sgn}(x)=2 \Theta(x)-1$, and noting that $\frac{d}{d x} \Theta(x)=\delta(x)$ [49], it follows that $\frac{d}{d x} \operatorname{sgn}(x)=2 \delta(x)$. As a result, the two-body contribution $V_{2}$ is consistent with LiebLiniger contact interactions. Specifically,

$$
V_{2}=\frac{\hbar^{2}}{m} 2 g \sum_{i<j} \delta\left(x_{i j}\right)+\frac{g^{2} \hbar^{2}}{m} \frac{N(N-1)}{2},
$$

which is precisely the contact pseudopotential that describes $s$-wave scattering, plus an additional constant. We further note that the three-body potential reduces to

$$
V_{3}=\frac{\hbar^{2}}{m} g^{2} \frac{N(N-1)(N-2)}{6} .
$$

The constant contribution of $V_{2}$ and $V_{3}$ adds up precisely to (minus) the energy of the McGuire solution in free space, i.e.,

$$
E_{0}=-\frac{g^{2} \hbar^{2}}{m} \frac{N\left(N^{2}-1\right)}{6} .
$$

We look for the Hamiltonian with a ground state,

$$
\Psi_{0}=\exp \left(-\frac{m \omega}{2 \hbar} \sum_{i=1}^{N} x_{i}^{2}+g \sum_{i<j}\left|x_{i j}\right|\right),
$$


which represents a McGuire soliton embedded in a harmonic trap. In this case, the long-range contribution becomes

$$
V_{2 \mathrm{~L}}=-\hbar \omega g \sum_{i<j}\left|x_{i j}\right|
$$

and the Schrödinger equation in the presence of the trap reads

$$
\begin{aligned}
& {\left[\sum_{i=1}^{N}\left(-\frac{\hbar^{2}}{2 m} \frac{\partial^{2}}{\partial x_{i}^{2}}+\frac{1}{2} m \omega^{2} x_{i}^{2}\right)\right.} \\
& \left.\quad+g \sum_{i<j}\left(\frac{\hbar^{2}}{m} 2 \delta\left(x_{i j}\right)-\frac{m \omega}{\hbar}\left|x_{i j}\right|\right)\right] \Psi_{0} \\
& =\left(E_{0}+\frac{N \hbar \omega}{2}\right) \Psi_{0} .
\end{aligned}
$$

This Hamiltonian is precisely the Lieb-Liniger-Coulomb model that can be alternatively derived using the Polychronakos formalism [50] as shown in Ref. [51]. The model describes one-dimensional bosons subject to contact interactions as well as a long-range pairwise contribution. The latter can be thought of accounting for a one-dimensional repulsive Coulomb interaction for $g>0$ or an attractive gravitational potential for $g<0$ [52]. The ground state of (25) exhibits a rich variety of regimes that have been characterized in Ref. [51]. In addition to the bright soliton behavior in the attractive regime, the system behaves as an incompressible Laughlin-like fluid with a flat density profile for weak repulsion. The appearance of Friedel oscillations and the formation of a Wigner crystal take place when the strength of the repulsive interactions is further increased. Interestingly, the coupling strengths of the contact and long-range interaction terms are not independent, and in the limit of vanishing trapping frequency the long-range contribution is absent and one recovers the Lieb-Liniger model in free space. This is a general feature of the family of models (12).

\section{GAUSSIAN PAIR FUNCTION}

As a generalization of the McGuire bright soliton solution we consider

$$
f\left(x_{i j}\right)=\exp \left(g\left|x_{i j}\right|^{2}\right),
$$

allowing for an arbitrary sign of $g$. The case with $g<0$ describes a Gaussian pair function that is commonly used in Monte Carlo calculations for quantum fluids. The corresponding Bijl-Jastrow many-body wave function is the ground state of the Hamiltonian $\hat{H}_{0}$ in Eq. (6) in which the two-body term is

$$
V_{2}=\frac{\hbar^{2}}{m} g N(N-1)+\frac{\hbar^{2}}{m} 4 g^{2} \sum_{i<j}\left|x_{i j}\right|^{2} .
$$

For the Gaussian pair function, the three-body contribution admits an expression compatible with a two-body potential as

$$
V_{3}=\frac{\hbar^{2}}{m} g^{2}(2 N-4) \sum_{i<j}\left|x_{i j}\right|^{2},
$$

and thus $V_{2}+V_{3}=-E_{0}+\frac{\hbar^{2}}{m} 2 N g^{2} \sum_{i<j}\left|x_{i j}\right|^{2}$. Here, the ground-state energy in free space equals $E_{0}=$ $-g N(N-1) \frac{\hbar^{2}}{m}$, where we note the linear and quadratic scaling with $g$ and $N$, respectively. The full interaction potential is described by pairwise attractive quadratic terms of the interparticle distance. Upon embedding in a harmonic trap, the long-range contribution reads

$$
V_{2 \mathrm{~L}}=-2 \hbar \omega g \sum_{i<j}^{N}\left|x_{i j}\right|^{2},
$$

and the ground-state wave function

$$
\Psi_{0}=\exp \left(-\frac{m \omega}{2 \hbar} \sum_{i=1}^{N} x_{i}^{2}+g \sum_{i<j}\left|x_{i j}\right|^{2}\right)
$$

has energy $\mathcal{E}_{0}=\frac{N \hbar \omega}{2}-g N(N-1) \frac{\hbar^{2}}{m}$. By contrast, when the pair function $f\left(x_{i j}\right)$ is chosen as a hyper-Gaussian, $f\left(x_{i j}\right)=$ $\exp \left(g\left|x_{i j}\right|^{n}\right)$, the parent Hamiltonian $H_{0}$ in the homogeneous case involves nontrivial three-body interactions of the general form in Eq. (8).

\section{HYPERBOLIC PAIR FUNCTION}

Another relevant system is the one associated with the following choice of the pair correlation function,

$$
f\left(x_{i j}\right)=\sinh \left(x_{i j} / \ell\right)^{\lambda},
$$

which describes bosons for even $\lambda$. We note that $f\left(x_{i j}\right) \sim$ $\exp \left(\lambda\left|x_{i j}\right| / \ell\right)$ for large $x_{i j}$, so one can expect a similarity with the Lieb-Liniger models discussed. However, the hyperbolic $f\left(x_{i j}\right)$ includes a hard-core constraint as $f\left(x_{i j}=0\right)=0$ and grows faster with the interparticle distance than the pair function in the Calogero-Sutherland model, $f\left(x_{i j}\right)=\left|x_{i j}\right|^{\lambda}$. In addition, the wave-function first derivative at contact $x_{i j}=0$ is continuous in this model. In this case,

$$
\begin{gathered}
V_{2}=\frac{\lambda \hbar^{2} N(N-1)}{2 m \ell^{2}}+\frac{\hbar^{2}}{m \ell^{2}} \sum_{i<j} \frac{\lambda(\lambda-1)}{\sinh ^{2}\left(x_{i j} / \ell\right)}, \\
V_{3}=\frac{\lambda^{2} \hbar^{2} N(N-1)(N-2)}{6 m \ell^{2}} .
\end{gathered}
$$

The pairwise hyperbolic potential diverges at the origin, imposing the hard-core constraint between particles. At low density it decays exponentially with the interparticle distance, as the one in the Toda lattice $[1,53]$. For the ground state in free space $\Phi_{0}=\prod_{i<j} \sinh \left(x_{i j} / \ell\right)^{\lambda}$, the energy is $E_{0}=$ $-\lambda^{2} \frac{\hbar^{2}}{6 m \ell^{2}} N\left(N^{2}-1\right)$, which resembles that of a McGuire solution in free space; see (22). In the presence of a harmonic trap, the long-range contribution reads

$$
V_{2 \mathrm{~L}}=\hbar \lambda \omega \sum_{i<j}^{N} \frac{x_{i j}}{\ell} \operatorname{coth}\left(\frac{x_{i j}}{\ell}\right) .
$$

We note that $x \operatorname{coth} x \sim|x|$ for large $x$, as in the Lieb-LinigerCoulomb model (25), but being attractive, it behaves as a one-dimensional gravitational potential [52]. In addition, it is also continuous and effectively harmonic near the origin, i.e., $x \operatorname{coth} x=1+x^{2} / 3+O\left(x^{4}\right)$. The energy of the trapped 
ground state

$$
\Psi_{0}=\exp \left(-\frac{m \omega}{2 \hbar} \sum_{i=1}^{N} x_{i}^{2}\right) \prod_{i<j} \sinh \left(x_{i j} / \ell\right)^{\lambda}
$$

is $\mathcal{E}_{0}=\frac{N \hbar \omega}{2}-\lambda^{2} \frac{\hbar^{2}}{6 m \ell^{2}} N\left(N^{2}-1\right)$.

\section{ANHARMONIC EXTERNAL POTENTIALS}

The previous examples illustrate the validity of the framework put forward, in which a Hamiltonian of the form (6) with ground state (5) can be used to construct a Hamiltonian (12) involving a harmonic trap and generally long-range pairwise interactions, with ground state (10). This setting can be generalized to include anharmonic external potentials. To this end, we next consider the ground state of the Hamiltonian with confinement to be described by a state

$$
\Psi_{0}=\exp \left(\sum_{i=1}^{N} v\left(x_{i}\right)\right) \Phi_{0},
$$

where $v(x)$ is an arbitrary function of the coordinates. In this case, $\Psi_{0}$ is a solution of

$$
\left[\hat{H}_{0}+\sum_{i=1}^{N} V\left(x_{i}\right)\right] \Psi_{0}+\frac{\hbar^{2}}{m} e^{\sum_{i=1}^{N} v\left(x_{i}\right)} \sum_{i=1}^{N} v^{\prime}\left(x_{i}\right) \partial_{x_{i}} \Phi_{0}=0,
$$

where the one-body potential reads

$$
V(x)=\frac{\hbar^{2}}{2 m}\left[v^{\prime \prime}(x)+v^{\prime}(x)^{2}\right] .
$$

Assuming $\Phi_{0}$ to have a Jastrow form as in Eq. (5), it follows that the parent Hamiltonian of the generalized ground state (36) is

$$
\begin{aligned}
{\left[\hat{H}_{0}\right.} & \left.+\sum_{i=1}^{N} V\left(x_{i}\right)\right] \Psi_{0} \\
& +\frac{\hbar^{2}}{m} \sum_{i<j}^{N}\left[v^{\prime}\left(x_{i}\right)-v^{\prime}\left(x_{j}\right)\right] \frac{f^{\prime}\left(x_{i j}\right)}{f\left(x_{i j}\right)} \Psi_{0}=0,
\end{aligned}
$$

with an energy eigenvalue $\mathcal{E}_{0}=0$. We note that for a specific form of $V(x)$, Eq. (38) can be integrated to find the function $v(x)$.

\section{QUANTUM SOLIDS}

An approach to characterize many-body states of quantum solids relies on pinning particles on a lattice, without relying on a tight-binding description, e.g., as done in the description of solid ${ }^{4} \mathrm{He}[54,55]$. Motivated by this approach, we next consider the family of Nosanov-Jastrow wave functions of the form

$$
\Psi_{0}=\prod_{i=1}^{N} \exp \left(-\frac{m \omega}{2 \hbar}\left(x_{i}-x_{i}^{0}\right)^{2}\right) \Phi_{0},
$$

where the Gaussian term localizes the $i$ th particle at a position $x_{i}^{0}$ and $\omega$ controls the tightness of the confinement. For instance, one can choose $x_{i}^{0}=i a$ where $a$ is the lattice spacing.
Two-particle correlations are encoded in $\Phi_{0}$ given by Eq. (5). The many-body Schrödinger equation (39) reduces to

$$
\left[\hat{H}_{0}+\sum_{i=1}^{N} \frac{m \omega^{2}}{2} \delta x_{i}^{2}-\hbar \omega \sum_{i<j}^{N} \delta x_{i j} \frac{f^{\prime}\left(x_{i j}\right)}{f\left(x_{i j}\right)}\right] \Psi_{0}=\frac{N \hbar \omega}{2} \Psi_{0},
$$

where the term in square brackets is the parent Hamiltonian of (40), with $\delta x_{i}=x_{i}-x_{i}^{0}$ and $\delta x_{i j}=\delta x_{i}-\delta x_{j}=x_{i j}$ $\left(x_{i}^{0}-x_{j}^{0}\right)$. Particles are thus confined by a lattice of harmonic oscillator wells and anharmonic effects can be included by considering a more general confining potential. We note that $\hat{H}_{0}$ is not altered and it is thus straightforward to introduce the quantum-solid Hamiltonians associated with the models discussed above. As an example, the quantum-solid version of the Calogero-Sutherland model is described by the Hamiltonian

$$
\begin{aligned}
\hat{H}= & \sum_{i=1}^{N}\left(-\frac{\hbar^{2}}{2 m} \frac{\partial^{2}}{\partial x_{i}^{2}}+\frac{m \omega^{2}}{2} \delta x_{i}^{2}\right) \\
& +\sum_{i<j}^{N}\left(\frac{\lambda(\lambda-1)}{\left|x_{i j}\right|^{2}}+\hbar \omega \lambda \frac{\delta x_{i j}^{0}}{x_{i j}}\right),
\end{aligned}
$$

which includes an additional Coulomb-like term, in which $\delta x_{i j}^{0}=x_{i}^{0}-x_{j}^{0}$ is constant for fixed site indices $i$ and $j$. Its ground state reads

$$
\Psi_{0}=\prod_{i=1}^{N} \exp \left(-\frac{m \omega}{2 \hbar}\left(x_{i}-x_{i}^{0}\right)^{2}\right) \prod_{i<j}\left|x_{i j}\right|^{\lambda},
$$

with energy eigenvalue $\mathcal{E}_{0}=\frac{N \hbar \omega}{2}[1+\lambda(N-1)]$. For $\lambda=1$ one recovers the hard-core bosonic pair correlation function. Due to the lattice structure, permutation symmetry is explicitly broken as the $i$ th particle is localized in the $i$ th harmonic well, but it can be restored by explicit symmetrization, after which the the ground-state wave function becomes

$$
\Psi_{0}=\left[\sum_{P \in S_{n}} \prod_{i=1}^{N} \exp \left(-\frac{m \omega}{2 \hbar}\left(x_{i}-x_{P(i)}^{0}\right)^{2}\right)\right] \prod_{i<j}\left|x_{i j}\right|^{\lambda},
$$

where the sum runs over the $N$ ! permutations of the $N$ lattice sites and $S_{N}$ denotes the symmetric group. In this case, $\Psi_{0}$ with $\lambda=1$ describes a Tonks-Girardeau gas in a lattice of harmonic wells.

\section{SUMMARY}

We have introduced an exact mapping between the ground state of a Hamiltonian in free space and the ground state of a dual Hamiltonian in the presence of a one-body trapping potential and additional many-body interactions. Whenever the homogeneous ground state takes the Bijl-Jastrow form, the dual Hamiltonian can be expressed in terms of the homogeneous one, supplemented with the one-body potential and two-body long-range interactions. This mapping can be used by fixing the pair correlation function entering the Bijl-Jastrow form, as we have done to find trapped states 
in systems with inverse-square, contact, quadratic, and Toda-like interactions. As an alternative, the functional form of the interparticle interactions can be first established, from which the pair function can be determined by integration. In addition, we have also shown how this family of Hamiltonians can be generalized to anharmonic external potentials and the description of quantum solids with ground states of Nosanov-Jastrow form.

Owing to this user-friendly construction we have identified unexplored examples of quasisolvable models. Among them, we have introduced a Lieb-Liniger-Coulomb model of onedimensional bosons subject to contact and gravitational or Coulomb interactions, the ground state of which is a McGuire soliton in a harmonic trap. Another instance resulting from the choice of a hyperbolic pair correlation function leads to trapped bosons with hard-core Toda-like interactions.

Our results should be broadly applicable in the quest for different beautiful models, describing (quasi)solvable and integrable quantum many-body systems. Possible extensions include systems in higher spatial dimensions [36,56], mixtures of multiple species [13,14], particles with an internal structure (e.g., spinors) [57], and their variants with a truncated interaction range [27-29,32,33]. Excited states in these systems can be explored by established techniques [1]. Our results should also find applications in the study of bright and dark trapped quantum many-body solitons, and quantum solids.

\section{ACKNOWLEDGMENTS}

It is a pleasure to acknowledge discussions with Gregory Astrakarchik, Mathieu Beau, Francesco Calogero, Íñigo L. Egusquiza, Xi-Wen Guan, Avinash Khare, and Suzanne Pittman.
[1] B. Sutherland, Beautiful Models (World Scientific, Singpaore, 2004).

[2] V. E. Korepin, N. M. Bogoliubov, and A. G. Izergin, Quantum Inverse Scattering Method and Correlation Functions (Cambridge University Press, Cambridge, U.K., 1997).

[3] M. Takahashi, Thermodynamics of One-Dimensional Solvable Models (Cambridge University Press, Cambridge, U.K., 1999).

[4] M. Gaudin, in The Bethe Wavefunction, edited by J.-S. Caux (Cambridge University Press, Cambridge, U.K., 2014).

[5] M. Gaudin, Phys. Rev. A 4, 386 (1971).

[6] M. T. Batchelor, X. W. Guan, N. Oelkers, and C. Lee, J. Phys. A: Math. Gen. 38, 7787 (2005).

[7] F. Calogero, J. Math. Phys. 12, 419 (1971).

[8] B. Sutherland, J. Math. Phys. 12, 246 (1971).

[9] M. Girardeau, J. Math. Phys. 1, 516 (1960).

[10] M. D. Girardeau, E. M. Wright, and J. M. Triscari, Phys. Rev. A 63, 033601 (2001).

[11] M. D. Girardeau, Phys. Rev. Lett. 97, 100402 (2006).

[12] A. del Campo, Phys. Rev. A 78, 045602 (2008).

[13] H. Ujino, A. Nishino, and M. Wadati, Phys. Lett. A 249, 459 (1998).

[14] M. D. Girardeau and A. Minguzzi, Phys. Rev. Lett. 99, 230402 (2007).

[15] A. Kundu, Phys. Rev. E 79, 015601(R) (2009).

[16] S. Giorgini, L. P. Pitaevskii, and S. Stringari, Rev. Mod. Phys. 80, 1215 (2008).

[17] S. Tan, arXiv:cond-mat/0412764.

[18] F. Werner and Y. Castin, Phys. Rev. A 74, 053604 (2006).

[19] Y. Castin and F. Werner, The unitary gas and its symmetry properties, in The BCS-BEC Crossover and the Unitary Fermi Gas, edited by W. Zwerger (Springer, Berlin, 2012), p. 127.

[20] N. Kawakami, J. Phys. Soc. Jpn. 62, 4163 (1993).

[21] N. Gurappa, A. Khare, and P. K. Panigrahi, Phys. Lett. A 244, 467 (1998).

[22] N. Gurappa and P. K. Panigrahi, Phys. Rev. B 59, R2490 (1999).

[23] K. Vacek, A. Okiji, and N. Kawakami, J. Phys. A: Math. Gen. 27, L201 (1994).
[24] N. Kawakami, Prog. Theor. Phys. 91, 189 (1994).

[25] K. Sogo, J. Phys. Soc. Jpn. 63, 879 (1994).

[26] K. Sogo, J. Phys. Soc. Jpn. 65, 3097 (1996).

[27] S. R. Jain and A. Khare, Phys. Lett. A 262, 35 (1999).

[28] G. Auberson, S. R. Jain, and A. Khare, J. Phys. A: Math. Gen. 34, 695 (2001).

[29] B. Basu-Mallick and A. Kundu, Phys. Lett. A 279, 29 (2001).

[30] M. Ezung, N. Gurappa, A. Khare, and P. K. Panigrahi, Phys. Rev. B 71, 125121 (2005).

[31] A. Enciso, F. Finkel, A. González-López, and M. Rodríguez, Phys. Lett. B 605, 214 (2005).

[32] S. M. Pittman, M. Beau, M. Olshanii, and A. del Campo, Phys. Rev. B 95, 205135 (2017)

[33] T. R. Tummuru, S. R. Jain, and A. Khare, Phys. Lett. A 381, 3917 (2017).

[34] A. Kundu, Phys. Rev. Lett. 83, 1275 (1999).

[35] M. T. Batchelor, X.-W. Guan, and N. Oelkers, Phys. Rev. Lett. 96, 210402 (2006).

[36] F. Calogero and C. Marchioro, J. Math. Phys. 14, 182 (1973).

[37] T. Kinoshita, T. Wenger, and D. S. Weiss, Science 305, 1125 (2004).

[38] B. Paredes, A. Widera, V. Murg, O. Mandel, S. Fölling, I. Cirac, G. V. Shlyapnikov, T. W. Hänsch, and I. Bloch, Nature (London) 429, 277 (2004).

[39] F. D. M. Haldane, Phys. Rev. Lett. 67, 937 (1991).

[40] Y.-S. Wu, Phys. Rev. Lett. 73, 922 (1994).

[41] M. V. N. Murthy and R. Shankar, Phys. Rev. Lett. 73, 3331 (1994).

[42] E. H. Lieb and W. Liniger, Phys. Rev. 130, 1605 (1963).

[43] E. H. Lieb, Phys. Rev. 130, 1616 (1963).

[44] M. Olshanii, Phys. Rev. Lett. 81, 938 (1998).

[45] J. B. McGuire, J. Math. Phys. 5, 622 (1964).

[46] M. Ishikawa and H. Takayama, J. Phys. Soc. Jpn. 49, 1242 (1980).

[47] J. Sato, R. Kanamoto, E. Kaminishi, and T. Deguchi, Phys. Rev. Lett. 108, 110401 (2012).

[48] J. Sato, R. Kanamoto, E. Kaminishi, and T. Deguchi, New J. Phys. 18, 075008 (2016). 
[49] R. N. Bracewell, The Fourier Transform and its Applications, 3rd ed. (McGraw-Hill, New York, 2000).

[50] A. P. Polychronakos, Phys. Rev. Lett. 69, 703 (1992).

[51] M. Beau, S. M. Pittman, G. E. Astrakharchik, and A. del Campo, arXiv:2009.03319.

[52] A. Muriel, Phys. Lett. A 56, 343 (1976).

[53] M. Toda, J. Phys. Soc. Jpn. 22, 431 (1967).
[54] C. Cazorla and J. Boronat, J. Phys.: Condens. Matter 20, 015223 (2007).

[55] C. Cazorla, G. E. Astrakharchik, J. Casulleras, and J. Boronat, New J. Phys. 11, 013047 (2009).

[56] A. Khare and K. Ray, Phys. Lett. A 230, 139 (1997).

[57] M. Girardeau, H. Nguyen, and M. Olshanii, Opt. Commun. 243, 3 (2004). 